FORMATION Formation emploi

Revue française de sciences sociales

125 | Janvier-Mars 2014

Pêle-Mêle

\title{
Les facteurs de risques psycho-sociaux chez les personnels de l'enseignement et de la recherche
}

Psychosocial risk factors with the staffs of education and research

Psychosoziale Risikofaktoren bei Beschäftigten im Lehramt und in der Forschung

Los factores de riesgos psicosociales entre el personal de docencia e investigación

Laurence Durat et Florence Brunet

\section{OpenEdition}

Journals

Édition électronique

URL : http://journals.openedition.org/formationemploi/4139

DOI : 10.4000/formationemploi.4139

ISSN : 2107-0946

Éditeur

La Documentation française

Édition imprimée

Date de publication : 25 mars 2014

Pagination : $29-46$

ISSN : 0759-6340

\section{Référence électronique}

Laurence Durat et Florence Brunet, «Les facteurs de risques psycho-sociaux chez les personnels de l'enseignement et de la recherche », Formation emploi [En ligne], 125 | Janvier-Mars 2014, mis en ligne le 31 mars 2016, consulté le 30 octobre 2020. URL : http://journals.openedition.org/formationemploi/ 4139 ; DOl : https://doi.org/10.4000/formationemploi.4139 


\title{
Les facteurs de risques psycho-sociaux chez les personnels de l'enseignement et de la recherche
}

\author{
FLoRENCE BRUNeT \\ Psychologue du travail. \\ Direction des Ressources Humaines, université de Haute-Alsace. \\ A participé au recueil et au traitement des données.
}

Laurence Durat

Enseignante-chercheure au Laboratoire Inter-universitaire des Sciences de l'Education et de la Communication (LISECEA 2310), université de Haute-Alsace.

Résumé

Les facteurs de risques psycho-sociaux chez les personnels de l'enseignement et de la recherche

Au-delà du champ habituel de la santé et de la sécurité, de l'aménagement des lieux de travail, la notion de risque professionnel a été étendue à une conception plus globale de la santé, comprenant la santé mentale. Nous nous intéressons ici à une université française qui a initié une évaluation des risques et des facteurs de risques psycho-sociaux. Il ressort de nos résultats que, pour la vitalité des collectifs de travail et la santé des individus, l'université doit rendre possibles des espaces d'échange sur les pratiques, des communautés discursives.

Mots-clés : université, enseignant, santé au travail, évaluation

Abstract

Psychosocial risk factors with the staffs of education and research

Beyond the usual field of health and security, the arrangement ok workplaces, we admitted the extension of the notion of professional risk in a more global conception of health, including mental health. To inform this problem we shall show the case of a French university which threw an assessment of psychosocial risks and risks factors. It is clear from our results that, for the vitality of collective work and the health of individuals, the university must make possible for exchange on practices, discursive communities.

Keywords: university, teacher, occupational health, evaluation

Journal of Economic Literature: I 23, M 12

Traduction : Auteurs 
Le travail est un facteur majeur de construction identitaire et d'équilibre social, comme l'ont montré Baudelot et Gollac (2003) ; cependant, il peut contribuer avec la même force au mal-être et à la fragilisation de la santé mentale des salariés. Aux préoccupations plus anciennes de sécurité et de santé au travail (aménagements des postes, conditions de travail, charge mentale, pathologies de surcharge, maladies professionnelles, stress) se sont ajoutées plus récemment des mises en cause des organisations quant à la pénibilité du travail, à l'épuisement professionnel, voire au harcèlement moral qu'il peut générer. L'obligation d'évaluation et de prévention faite à l'employeur a mis la question des risques psychosociaux au centre des débats dans le secteur privé, en lien avec la mise en cause de nouveaux types de management par la performance; dans le secteur public où cette obligation existe depuis 2009, les changements ont été très profonds également, bien que peu questionnés (Abord de Chatillon \& Desmarais, 2012).

Des réformes majeures transforment le paysage universitaire, avec des objectifs affichés de rationalisation des moyens et des offres de formation comme de la recherche, et une autonomisation accrue des établissements combinée à une pression aux regroupements régionaux (politique de sites). Dans ce contexte de plus en plus contraint avec une tendance généralisée aux restrictions de moyens, les perspectives d'avenir offertes au personnel de l'enseignement supérieur suscitent de nombreuses inquiétudes. Comment se situent-ils dans cette représentation mouvante et recomposée qui leur est proposée ? Que faire de cette nouvelle université qui émerge ? Des questions immédiates et de plus long terme se posent à eux sans préparation, sans que l'on sache très bien comment ils s'en saisissent.

Nous visons ici une meilleure compréhension des facteurs de risques psycho-sociaux et nous privilégierons une entrée par le travail, et non par la souffrance. Nous faisons l'hypothèse qu'un déficit de ressources pour le travail collectif s'ajoute à des pressions accrues liées à la modification de l'environnement de travail pour fragiliser les personnels universitaires. Afin de documenter cette problématique, nous présenterons d'abord quelques éléments de contexte et le cadre théorique; nous verrons ensuite comment l'évaluation a été conçue pour une université de taille moyenne ; enfin, nous présenterons les résultats obtenus ; ainsi, pour toutes les catégories de personnel, le risque premier est lié à la souffrance et au malêtre ressentis ; pour les personnels d'enseignement et de recherche, les facteurs de risques sont majoritairement liés au management et aux relations de travail puis aux exigences et à l'organisation du travail. Ce qui pose la question du manque d'espaces de délibération sur les pratiques de travail au sein de l'université.

\section{Contexte et cadre théorique: une problématique qui répond à une demande sociale}

Les débats autour du terme " risques psycho-sociaux » (RPS) montrent l'écho politique et social de cette problématique qui semble répondre à une demande sociale (Gollac, 2011) 
diversement abordée selon les disciplines ou courants (psychiatrie, médecine du travail, sociologie, psychologie du travail et ergonomie, psychopathologie du travail). Critiquée pour la « sanitarisation » ou pour la judiciarisation des relations de travail qu'elle est susceptible d'entraîner (Lhuilier, 2012), la notion de RPS peut donner lieu à des visions réductrices qui présentent l'inconvénient de placer hors du champ de l'organisation le traitement de ses causes.

Nous verrons d'abord que la notion de RPS est le fruit d'une évolution conceptuelle, que le contexte universitaire étudié est soumis à de profondes restructurations, et distinguerons les risques des troubles et des facteurs de risques.

\subsection{Les risques psycho-sociaux : une notion en évolution}

Depuis les travaux de Marie-France Hirigoyen qui ont permis, en France, de faire émerger la notion de harcèlement moral et de violence psychologique (1998, 2001), d'identifier ses effets délétères (souffrance, dépression, stress) et sa prise en compte dans le code du Travail en 2002 (L1152-1), la dimension psychique du mal-être lié au travail est reconnue et abordée dans divers champs disciplinaires qui se donnent pour objet les travailleurs pris individuellement ou collectivement.

La problématique des risques psycho-sociaux (RPS) s'est ensuite développée. Elle a conduit à des prises de conscience, d'abord dans les entreprises privées après une vague de suicides au travail qui ont fait l'objet d'une forte médiatisation : les cas de France Télécom, du Technocentre de Renault, de la centrale nucléaire de Chinon en particulier, ont jeté une lumière nouvelle sur la manière de concevoir la santé au travail.

Cette prise en compte a été suscitée par les nombreuses études menées sur les risques professionnels (INVS, 2004 ; 2006 ; 2009 ; Sumer, 1994 ; 2003 ; 2010 ; Sahler \& al., 2007 ; INRS, 2006 ; 2010). Elle s'est aussi traduite par la volonté de l'Etat d'en faire une priorité avec le lancement, en 2009, du Plan d'action d'urgence au travail pour mobiliser les employeurs des secteurs privé et public.

Depuis la signature de l'Accord Santé et Sécurité au travail dans la fonction publique (2009), les risques psycho-sociaux se sont également imposés comme une préoccupation majeure dans le domaine de la santé au travail dans le secteur public.

Ainsi, l'employeur doit prendre les mesures nécessaires pour assurer la sécurité et protéger la santé physique et mentale des travailleurs (L 4121-1), c'est-à-dire engager des actions d'évaluation des risques professionnels et de la pénibilité du travail, des actions d'information et de formation ainsi que la mise en place d'une organisation et de moyens adaptés. Il doit également veiller à l'adaptation de ces mesures pour tenir compte des transformations $d u$ contexte et tendre à l'amélioration des situations existantes repérées.

La psycho-dynamique du travail et la clinique de l'activité tentent d'aborder cette question sous l'angle de l'activité de travail, afin de «dépsychologiser» cette approche et de faire toute 
sa place à la dimension sociale et organisationnelle des RPS. Van Belleghem \& al. (2013) rappellent, à cet égard, que le psychosocial n'est pas un risque en soi, mais une dimension présente dans toute situation de travail et de vie, et avant tout une ressource pour créer, innover, produire. Selon Volkoff (2013), les évolutions générales de l'organisation du travail réduisent la stabilité et l'homogénéité des collectifs de travail. Elles impliquent aussi un réaménagement des stratégies de travail de chacun, ce contexte étant potentiellement porteur de problèmes graves. Il s'agit selon Clot (2010) de ne pas se focaliser sur les situations d'urgences individuelles mais de mettre en discussion le travail afin de redonner aux professionnels un "pouvoir d'agir" de nature à traiter les causes et non les symptômes d'insatisfaction au travail.

Nous retiendrons ici la définition issue de la synthèse de Gollac \& al. (2011, p. 31) pour qui les risques psycho-sociaux sont : "les risques pour la santé mentale, physique et sociale, engendrés par les conditions d'emploi et les facteurs organisationnels et relationnels susceptibles d'interagir avec le fonctionnement mental ."

\subsection{Un contexte universitaire marqué par des restructurations}

La question des changements organisationnels et des restructurations est en effet importante, de même que les exigences et les modes d'organisation du travail ; en effet, aucune décision structurante ne peut être détachée de ses répercussions sur la santé physique et psychique des salariés.

Les orientations suivies par les politiques de l'enseignement supérieur et de la recherche françaises convergent avec celles des autres pays européens depuis près de trente ans (Musselin, 2009). Il s'agit de renforcer l'autonomie des établissements et de leurs exécutifs et de s'inscrire dans une compétition vers l'excellence scientifique qui s'adosse à des évaluations nationales et internationales. Les transformations sont profondes : la loi sur l'autonomie des universités de 2007, avec le passage aux responsabilités et compétences élargies (RCE) en matière financière et de ressources humaines, et les nouveaux modes de gouvernance des établissements, les regroupements des universités et organismes en grands pôles, le financement par projet et globalement l'imprévisibilité dans laquelle évoluent les organisations, ont transformé l'enseignement supérieur et la recherche en France ; le périmètre d'intervention, les modes de conception et de réalisation de l'activité et les conditions de travail des professionnels de l'université sont impactés directement.

Plusieurs universités françaises ont initié des démarches distinctes d'évaluation des risques. Nous rendrons compte ici d'une démarche d'évaluation des risques et des facteurs de risques menée, de janvier à juin 2013, dans une université de l'Est de la France composée d'un millier de personnes (hors vacataires) pour un effectif de 8000 étudiants en moyenne. Cette université est soumise à la pression aux regroupements insufflée par le ministère de l'Enseignement supérieur et de la Recherche. Elle se rattache (sans fusionner), par convention et en même temps que d'autres établissements (écoles d'ingénieurs notamment), à 
un site piloté par une grande université régionale. Les décisions génèrent débats et inquiétudes mais ont été approuvées par les instances internes. Le site requiert un pilotage conjoint entre les différents établissements, chacun conservant son autonomie de gestion ; en interne, l'équipe présidentielle a opté pour un " fédéralisme " fondé sur l'élaboration de consensus entre les composantes. Les relations sociales apparaissent bien établies et apaisées dans cet établissement.

\subsection{L'évaluation des effets et des causes : entre facteurs de risques et troubles}

L'Anact (Association nationale pour l'amélioration des conditions de travail) souligne la pluri-factorialité des risques psycho-sociaux caractérisant à la fois l'organisation du travail et les relations interindividuelles. Elle distingue les "risques" (probabilité d'y être confronté) des "troubles" (conséquence d'une exposition aux risques). Les troubles peuvent se manifester sous différentes formes : le stress, l'épuisement professionnel (burn-out), la dépression, la décompensation, et diverses pathologies ayant pour origine l'environnement professionnel. Enfin, ces troubles proviennent de causes, les "facteurs" de risques : les violences (verbales ou physiques) externes (provenant de personnes non employées par l'établissement) ou internes (entre collègues ou de la part du supérieur hiérarchique), le harcèlement moral ou sexuel, les discriminations en particulier. Les facteurs psycho-sociaux désignent les interactions entre la personne au travail et les caractéristiques du travail et de son environnement (les dimensions organisationnelles et relationnelles du travail). Cette définition rappelle aussi que la santé psychique n'est pas seulement une dynamique individuelle, mais qu'elle se construit dans la relation aux autres (par la reconnaissance, la possibilité d'échanges et de coopération dans le travail, avec le soutien des collègues et de la hiérarchie).

S'agissant des RPS, il est demandé aux responsables de rechercher en quoi le cadre de travail de leurs collaborateurs est susceptible de générer des RPS. Les facteurs à l'origine de la plupart des risques identifiés sont classiquement regroupés en quatre grandes familles :

- les exigences du travail et son organisation ;

- le management et les relations de travail ;

- la prise en compte des valeurs et attentes des personnels ;

- les changements du travail. 


\section{Encadré 1 : Méthodologie retenue}

Quand l'évaluation des risques psychosociaux (RPS) est menée auprès du personnel uniquement par voie de questionnaire, nécessairement anonyme, les résultats obtenus permettent de mesurer de grandes tendances mais pas de cibler la nature, les raisons, les voies d'amélioration possible des risques encourus.

Or les RPS représentent un objet qui reste difficile à appréhender pour les organisations ; dès lors, si la démarche permet de détecter un climat détérioré sans pouvoir pointer plus finement les dysfonctionnements, on ne peut que formuler des actions de prévention elles-mêmes généralistes (formation au management, gestion du stress) dont les répercussions sur le vécu des salariés restent très hypothétiques. C'est l'écueil actuel de nombreuses démarches effectuées en entreprise, qui pourtant ont mis en œuvre des moyens considérables sans aboutir pour autant à des résultats satisfaisants (cas de Renault dont 37000 tests ont été inadéquats pour discerner les sources de tensions, Sahler \& al., 2007). Certes, l'évaluation permet de se mettre en conformité avec les injonctions réglementaires mais elle échoue à produire un cadre pertinent d'amélioration sur la longue durée. La raison principale échoit à ce que Molinier (2011, p. 15) nomme « la sempiternelle réitération du déni du travail » lorsque les mesures des troubles sont complètement détachées des références concrètes à l'activité.

Nous avons donc choisi une méthodologie qualitative qui s'adosse à ce que les personnes disent et pensent du contenu de leur travail, en poursuivant un double objectif : ouvrir l'évaluation à la dimension collective du travail et recueillir des énonciations quant aux facteurs de risques afin de fonder le plan de prévention sur le travail vécu. Cela nous a amené à préférer la méthode des entretiens collectifs (Froment, 2012) à celle des entretiens individuels. Dans une phase ultérieure, un questionnaire a repris les thématiques identifiées sur le terrain pour en mesurer le poids dans la population totale.

Conformément au choix effectué par le groupe de travail du CHSCT (Comité d'hygiène, de sécurité et des conditions de travail) de l'établissement, les entretiens collectifs ont été proposés par catégorie de personnel. II s'agissait d'aborder des thématiques propres aux périmètres des personnes sollicitées et aussi d'éviter des comportements d'inhibition et de distance sociale ; au total, huit entretiens collectifs ont été organisés entre janvier et avril 2013. Chaque entretien visait les personnels administratifs et techniques de niveau $A$ (fonctions d'encadrement), de niveau B (fonctions d'application), de niveau C (fonctions d'exécution), les personnels exerçant des fonctions de direction, les enseignants du second degré (professeurs agrégés ou PRAG professeurs certifiés ou PRCE, professeurs associés à temps partiel ou PAST, enseignants contractuels), les enseignants-chercheurs, les professeurs d'université, enfin les doctorants, post-doctorants, et associés temporaires à l'enseignement et à la recherche, ou ATER (Attaché temporaire d'enseignement et de recherche) et enfin les lecteurs.

L'invitation à participer aux groupes focus s'est effectuée en respectant une représentation des différentes composantes de l'université pour la sélection des participants (30 personnes sollicitées pour chaque entretien collectif) avec libre choix (l'échantillon représenté est différent de l'échantillon sollicité). La consigne de départ, après une brève présentation de la démarche et une définition des RPS, se limitait à une invitation à exprimer leur avis sur leurs satisfactions et insatisfactions concernant le travail. Des reformulations étaient parfois soumises au groupe ainsi que des demandes d'éclaircissement quant au sens à accorder aux énonciations; de même que des invitations à préciser les affects provoqués par certaines situations (du type " et qu'avezvous ressenti, à ce moment-là ? ») afin de préciser le lien entre description et trouble provoqué. 


\section{Suite encadré 1}

En fin d'entretien, si cela n'avait pas été spontanément abordé, une question était posée, concernant la manière dont les personnes envisageaient leur avenir dans l'établissement.

Globalement, 63 personnes ont ainsi été entendues dans les entretiens d'une durée de 90 à 150 minutes. Ces entretiens ont été animés par le même binôme, composé d'une psychologue du travail et d'une enseignante-chercheure, afin d'homogénéiser les conditions de recueil des données. Les entretiens ont été enregistrés et retranscrits, la catégorisation a été faite par analyse inductive de contenu, par entretien et par chaque analyste, puis comparée à la catégorisation effectuée par le deuxième analyste pour faire émerger les catégories et sous-catégories retenues. Ainsi seules les grandes familles de facteurs de risques ont été conservées pour la classification du corpus, les autres éléments de catégorisation ayant été induits à partir des énonciations des participants. Afin de faciliter l'analyse et pour comparer les différentes catégories, chaque catégorie et sous-catégorie a été codée et une pondération a été attribuée en proportion du nombre d'énonciations par groupe focus et par thème.

\section{Résultats: \\ du mal-être aux violences}

Nous présenterons d'abord l'identification des risques psycho-sociaux perçus et nous verrons ensuite les facteurs de risques (tout l'échantillon puis plus particulièrement les personnels chargés d'enseignement et de recherche) tels qu’ils ressortent des énonciations recueillies.

\subsection{Des troubles et risques variables selon le statut}

Sur un total de 526 énonciations relatives aux troubles et risques psycho-sociaux (sur 1652 énonciations en tout), il apparaît principalement les états de souffrance ou malêtre (323) et secondairement de fatigue et de stress (135) ; de façon moindre, de démotivation (53), puis de violences (12) et de déséquilibre entre vie professionnelle et vie privée (3). On retrouve cette distribution dans le graphique 1. 


\section{Graphique 1 : répartition des risques psycho-sociaux par catégorie de personnel et type de risques}

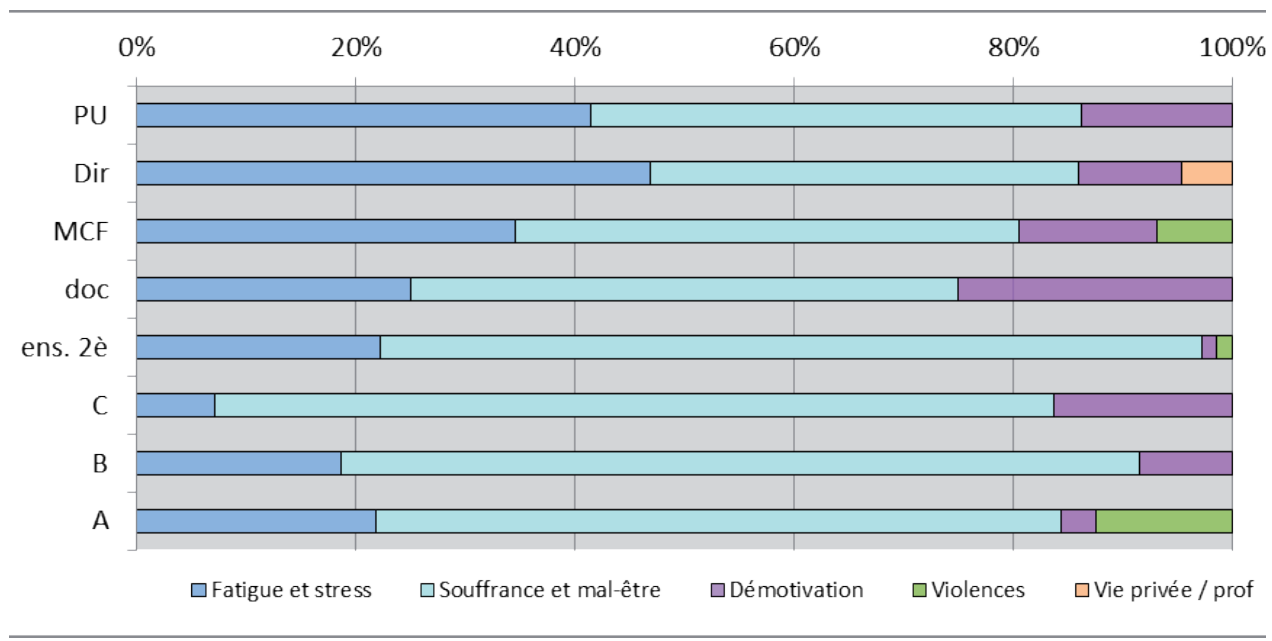

Sigles : PU : professeurs d'université ; Dir : directeurs; MCF : maîtres de conférences ; doc : doctorants, ATER : Attaché temporaire d'enseignement et de recherche, lecteurs ; 2 è : enseignants du second degré ; $A, B, C$ : personnel administratif et technique de catégorie $A, B, C$.

Exemple de lecture : Pour le personnel de catégorie $B$, plus de $20 \%$ des risques identifiés sont liés à la fatigue et au stress, près de $70 \%$ à une souffrance ou du mal-être, et moins de $10 \%$ à la démotivation.

Source : L. Durat, F. Brunet (2013), Rapport RPS au CHSCT.

Les enseignants du second degré apparaissent les plus sensibles aux risques de souffrance et mal-être, alors que les professeurs d'université expriment moins cette exposition. Les doctorants cumulent des expressions de mal-être avec une plus forte démotivation que les autres catégories.

Dans les groupes focus des personnels administratifs et techniques, une fois passée la surprise d'être consultés et entendus, la construction de consensus s'est faite de façon très comparable sur quelques points de cristallisation se rapportant à des énonciations de frustrations, d'impuissance, ou de révolte au regard du manque de considération quant à leur place dans l'institution. Pour les enseignants du second degré, la question du rattachement à différents types de composantes semblait jouer sur leur identité professionnelle, au-delà des différences disciplinaires ; de nombreuses plaintes portaient sur des conflits non réglés entre collègues. Parmi les enseignants-chercheurs, des conflits de valeurs et de conceptions de leurs rôles prédominaient, ces situations produisant des dissensus au sein même des entretiens collectifs. Les directeurs comme les professeurs d'université se plaignaient majoritairement d'une forte charge de travail, d'évaluations permanentes et de manque de visibilité sur l'avenir (tableau 1). Les doc- 
torants exprimaient leurs difficultés à se situer dans l'université et à se faire entendre. L'identification de clivages forts en fonction des statuts, de difficulté de communication (cloisonnement entre composantes) traversait tous les groupes focus.

Tableau 1 : extraits concernant les risques « souffrance et mal-être »

\begin{tabular}{|c|c|}
\hline Catg & Souffrance, mal-être \\
\hline C & $\begin{array}{l}\text { "Que tout le monde soit aligné, après c'est le système qui est ainsi fait que tout le monde soit sur une grille parce que tu es C tu } \\
\text { as tant. Mais ta collègue qui n'a aucune responsabilité va toucher autant que toi qui es responsable, qui plus est responsable de ta } \\
\text { collègue... à un moment donné...." }\end{array}$ \\
\hline C & « J'étais pilier du service selon l'entretien d'évaluation, à mon retour après un Zème enfant, je ne suis plus rien du tout.» \\
\hline B & $\begin{array}{l}\text { « On va vous dire : faut surtout pas prendre d'initiatives } \\
\text { J'ai eu le cas quand je suis arrivée à C. J'apportais une certaine expérience quand je proposais un travail : « ah mais on vous a } \\
\text { jamais demandé ça ... de quel droit vous vous permettez de prendre des initiatives?». C'était même dans mon entretien d'évalua- } \\
\text { tion.» }\end{array}$ \\
\hline B & $\begin{array}{l}\text { "Clivage entre les BIATSS et les enseignants. Nous les BIATSS, on est les pions; } y^{\prime} \text { a pas de dialogue ; y'a les tout puissants qui sont } \\
\text { au-dessus et nous en bas; on est content d'avoir un travail mais c'est vrai on subit tout » }\end{array}$ \\
\hline A & $\begin{array}{l}\text { «Par rapport à l'intégration... J'ai fait plusieurs postes dans d'autres établissements, quand on arrive ici il faut être opérationnel } \\
\text { t out de suite ; C'est pire. On a l'impression qu'on est sur la sellette, carrément. En même temps on se sent observée. Est-ce qu'elle } \\
\text { va convenir au poste? C'est vraiment un stress énorme. Les premiers temps où j'étais ici j'en dormais pas la nuit.» }\end{array}$ \\
\hline A & $\begin{array}{l}\text { "On peut parfois se sentir seul, une très grande solitude avec une grande responsabilité ; pas de conseil, pas de partage, } \\
\text { débrouilles-toi. » }\end{array}$ \\
\hline MCF & $\begin{array}{l}\text { «C'est vrai que ça fait mal quand y'a une personne qui vous passe devant ; vous aspirez à telle ou telle responsabilité, vous vous } \\
\text { investissez pour votre département, pour votre école ou pour votre université et que finalement on vous dit : « non le poste n'est } \\
\text { pas pour toi, il est pour l'autre là-bas. T'es un bon p'tit gars mais c'est comme ça... » }\end{array}$ \\
\hline MCF & $\begin{array}{l}\text { «Moi j’ai des élèves ingénieurs en 3è année de chimie qui ne connaissent pas la couleur du soufre ! Un chimiste bac + } 5 \text { qui ne } \\
\text { connaît pas la couleur du soufre, je suis navrée !... Et le pire, c'est que ça va devenir des ingénieurs chimistes qui vont piloter nos } \\
\text { centrales nucléaires ; j'ai peur!» }\end{array}$ \\
\hline 2e D & $\begin{array}{l}\text { «Les BIATOSS sont maltraités par leur hiérarchie, souvent on leur parle comme à des chiens ; les secrétaires, quand j'entends } \\
\text { comment on s'adresse à elles et après quand je vois le salaire qu'elles touchent pour le travail qu'elles font, c'est effrayant. » }\end{array}$ \\
\hline 2e D & «On nous traite de cons parce qu'on est professionnalisant » \\
\hline Dir & $\begin{array}{l}\text { "Je rejoins ce qui vient d'être dit et ça je l'ai reçu d'un certain nombre de collègues qui sont très inquiets, personnels aussi bien MCF } \\
\text { qu'administratifs, de ce qu'ils entendent des problèmes de fusion, de rationalisation des services et je parle même pas d'absorption. » }\end{array}$ \\
\hline Dir & $\begin{array}{l}\text { «On a fait ce travail, qu'est-ce que ça va devenir ? Je ne parle même pas des carrières personnelles ; y’a une réelle inquiétude sur : } \\
\text { finalement, tout ce qu'on a fait, est-ce que ça va être balayé d'un revers de main?» }\end{array}$ \\
\hline
\end{tabular}

Sigles : BIATOSS : bibliothécaires, ingénieurs, administratifs, techniciens, ouvriers de service et de santé ; BIATSS : Bibliothèques, ingénieurs, administratifs, techniciens de service et de santé.

PU : professeurs d'université ; Dir : directeurs ; MCF : maîtres de conférences ; doc : doctorants, ATER (attaché temporaire d'enseignement et de recherche), lecteurs ; 2è : enseignants du second degré ; $A, B, C$ : personnel administratif et technique de catégorie $A, B, C$. (respectivement cadre, encadrement intermédiaire, exécution).

Source : L. Durat, F. Brunet (2013) Rapport RPS au CHSCT, 


\subsection{Les facteurs de risques chez les chargés d'enseignement et de recherche}

Les 1317 énonciations concernant les facteurs de risques sont réparties dans ces quatre familles en fonction des groupes focus de personnel d'enseignement et de recherche dans lesquels elles ont été recueillies (proportions de base 1 ramenées à 0.6 pour la lisibilité des graphiques sous forme de radars).

Si l'on se réfère à la classification en quatre grandes familles de facteurs de risques, on constate que deux familles de facteurs de risques sont plus massivement représentées (graphique 2) ; il s'agit de la famille management, relations de travail (hiérarchie, encadrement ; relations ; équité, transparence ; information, communication ; intégration des nouveaux) puis de la famille exigences et organisation du travail (exigences du poste, des missions ; organisation du travail ; ressources).

\section{Graphique 2 : répartition des 4 facteurs de risques pour les 4 catégories}

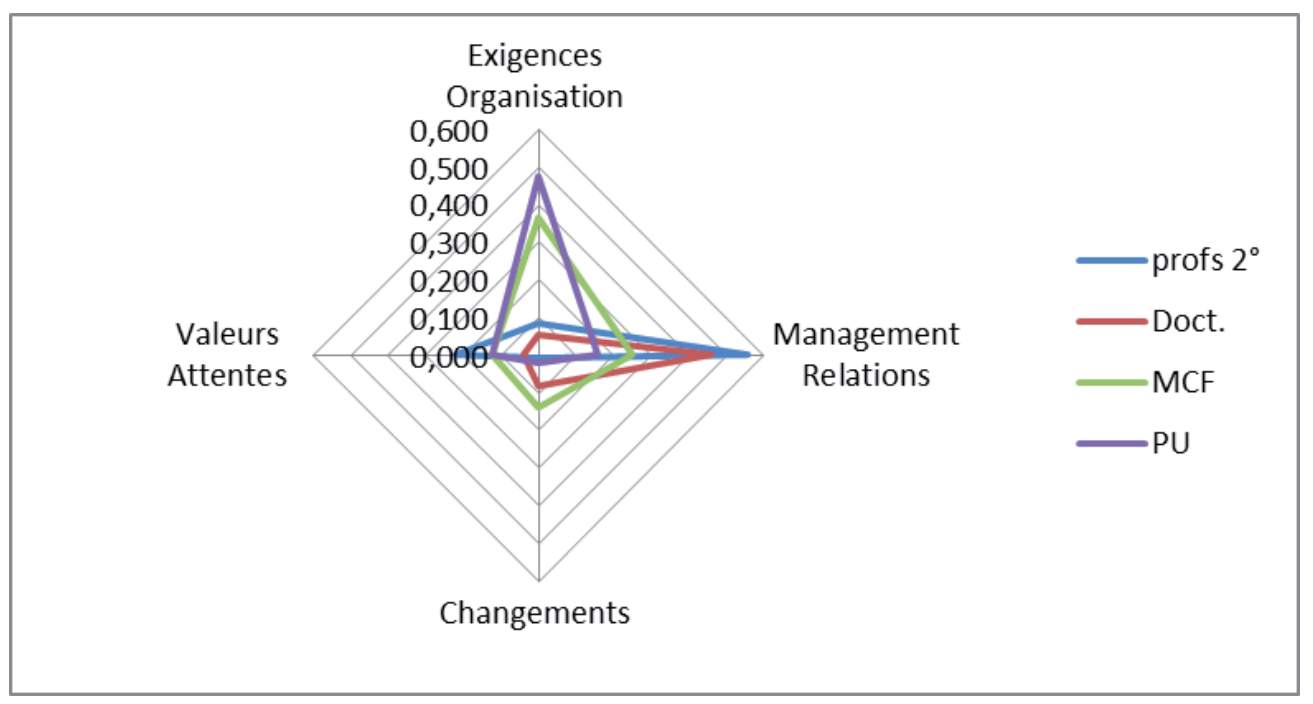

PU : professeurs d'université ; MCF : maîtres de conférences ; doc : doctorants, ATER : Attaché temporaire d'enseignement et de recherche ; lecteurs ; 2è : enseignants du second degré.

Exemple de lecture : pour les professeurs d'université (PU), près de $50 \%(0.47)$ des facteurs de risques sont liés aux exigences du poste et à l'organisation du travail.

Source : L. Durat, F. Brunet (2013) Rapport RPS au CHSCT. 
La distribution de ces facteurs présente cependant des profils très distincts, que l'on peut rapprocher deux par deux : d'une part, les deux catégories d'enseignants-chercheurs (MCF et PU), et d'autre part les enseignants du second degré (PRAG, PRCE, PAST, enseignants contractuels) et les contractuels de l'enseignement et de la recherche (doctorants, ATER et lecteurs). Pour les premiers, MCF et PU, la sensibilité aux facteurs de risques liés aux exigences et à l'organisation du travail prédomine, alors que les seconds se montrent plus sensibles au management et aux relations de travail.

Tous ces personnels sont amenés à travailler dans les mêmes composantes ; pourtant, ils ont été diversement impactés par les évolutions des conditions de travail liées au nouveau contexte de l'enseignement supérieur et de la recherche en France.

\subsubsection{Les enseignants-chercheurs : des risques liés aux évaluations et à l'organisation du travail}

Pour les enseignants-chercheurs, les conditions de réalisation de leur activité ont effectivement beaucoup changé. Deux points de crispation émergent dans les entretiens : la question de l'évaluation et la difficulté de concilier les deux missions (enseignement et recherche) distinctes, avec la prégnance de plus en plus forte des évaluations de leur production scientifique au niveau individuel (publications scientifiques) comme collectif (évaluation de leur laboratoire). Des recherches récentes identifient l'évaluation comme cause de souffrance (Dejours, 2003) ou montrent que celle-ci sert à l'intensification du travail, utilisée comme moyen d'obtenir un effort de tous en ne récompensant que quelques-uns (Gollac \& Volkoff, 2011).

Quelques exemples (tableau 2) illustreront les problèmes évoqués à l'origine d'insatisfactions, de dysfonctionnements, voire générant stress, mal-être et démotivation. Pour leur mission de recherche, les enseignants-chercheurs sont ainsi entrés dans une logique de justification (être ou ne pas être publiant) alors même qu'ils ne peuvent agir sur certains déterminants de la situation (une thématique de recherche qui est plus ou moins bien "reçue " par les milieux académiques, un laboratoire plus ou moins bien reconnu, l'existence d'une réelle dynamique de recherche, l'encadrement proposé, etc.). Dans les établissements, les tâches administratives et pédagogiques sont nombreuses et parfois contradictoires ; ils sont également sollicités, au-delà de leur service d'enseignement, pour participer au bon déroulement des formations. Les questions se posent alors à chaque enseignant-chercheur sous forme d'alternative, provoquant tensions entre leurs missions d'enseignement et de recherche et tentatives de compromis ou renoncement toujours problématiques (privilégier pendant un temps l'une ou l'autre des missions). 
Tableau 2 : problèmes concernant les exigences et l'organisation du travail

\begin{tabular}{|c|c|}
\hline $\begin{array}{l}\text { Exigences du poste, de la } \\
\text { mission } \\
\text { Difficultés liées à la recherche }\end{array}$ & $\begin{array}{l}\text { - difficultés liées aux charges administratives (manque de volontaires / charges non valori- } \\
\text { sées pour les EC); } \\
\text { - difficultés liées à la recherche de financements; } \\
\text { - difficultés pour recruter de bons thésards; } \\
\text { - manque de temps pour la recherche (à cause des charges administratives); } \\
\text { - multiplicité des missions; } \\
\text { - priorité à la recherche (au détriment de l'enseignement) ; } \\
\text { - de plus en plus de pression sur la recherche (difficultés variant avec le niveau de recon- } \\
\text { naissance du labo) ; } \\
\text { - problématique des EC qui ne font pas de recherche ; } \\
\text { - problématique des chercheurs isolés; } \\
\text { - problème du sur-encadrement de thésards par certains EC (confusion de rôles); } \\
\text { - difficultés liées à la publication scientifique (durée longue, pas d’aide); } \\
\text { - difficultés pour mener une thèse à son terme (doctorant comme encadrant). }\end{array}$ \\
\hline $\begin{array}{l}\text { Organisation du travail } \\
\text { Multiplicité et pertinence des } \\
\text { évaluations, des indicateurs, des } \\
\text { contrôles }\end{array}$ & $\begin{array}{l}\text { - lourdeur des démarches et caractère obligatoire des enquêtes / formations; } \\
\text { - inflation des évaluations et du « rendre compte »; } \\
\text { - doutes sur la pertinence et l'utilité des enquêtes, des évaluations. }\end{array}$ \\
\hline
\end{tabular}

EC : enseignants-chercheurs.

Source : L. Durat, F. Brunet (2013) Rapport RPS au CHSCT.

La pression liée aux attentes institutionnelles est donc fortement ressentie et rétroagit bien évidemment sur les choix professionnels et sur la vie collective (prendre la responsabilité d'une formation rend service à l'institution mais nuit à la capacité de recherche de enseignants-chercheurs). Cette évolution signale un risque de plus grande individualisation (à travers l'évaluation notamment), de diversification des tâches et des charges accrues qui ne sont que peu soutenues au plan organisationnel, ni en termes d'accompagnement, ni en termes de formation. Elle génère donc de fortes inquiétudes (modulation des services pour les enseignants-chercheurs, évolutions à venir concernant les carrières, perte d'autonomie) et le sentiment de non-reconnaissance de l'engagement des personnels, source d'insatisfactions voire de mal-être, exacerbé par les exigences organisationnelles, comme le pointe Viry (2006). Or les formes d'organisations réputées pathogènes sont non seulement caractérisées par une forte pression, mais aussi par une faible autonomie, un faible soutien (Karasek, Theorell, 1990) et peu de reconnaissance (Siegrist et al., 2004). On retrouve ces tensions exprimées en termes de valeurs et attentes insatisfaites (tableau 3). 
Tableau 3 : problèmes concernant les valeurs et attentes

\begin{tabular}{|c|c|}
\hline $\begin{array}{l}\text { Ethique } \\
\text { Conflits de valeurs }\end{array}$ & $\begin{array}{l}\text { - déconstruction d'une formation à chaque évaluation ; } \\
\text { - devoir renoncer à une responsabilité par manque de reconnaissance ; } \\
\text { - s'approprier des publications (excès de co-signatures de publications pour certains cher- } \\
\text { cheurs); } \\
\text { - conflits pour s'approprier des heures d'enseignement ; } \\
\text { - certains choix sont des freins (charges administratives pour les EC); } \\
\text { - ne pas avoir le sentiment de travailler à un collectif, à une œuvre commune. }\end{array}$ \\
\hline $\begin{array}{l}\text { Reconnaissance } \\
\text { Manque de reconnaissance }\end{array}$ & $\begin{array}{l}\text { - investissement, conscience professionnelle non récompensés; } \\
\text { - manque de reconnaissance pour les MCF qui ont des responsabilités pédagogiques; } \\
\text { - aucune valorisation des compétences; } \\
\text { - certaines filières sont mises en avant comme faire-valoir, aucune reconnaissance concrète ; } \\
\text { - manque de reconnaissance au niveau de la recherche industrielle appliquée (contrats mais } \\
\text { pas de publications); } \\
\text { - manque de reconnaissance de l'investissement collectif au détriment de l'investissement } \\
\text { individuel ; } \\
\text { - reconnaissance au niveau de la composante mais pas au niveau de l'Université ; } \\
\text { - pour certains projets, manque de reconnaissance des MCF par rapport aux PU. }\end{array}$ \\
\hline
\end{tabular}

Sigles: $E C$ : enseignants-chercheurs; $P U$ : professeurs d'université; $M C F$ : maîtres de conférences.

Source : L. Durat, F. Brunet (2013) Rapport RPS au CHSCT.

En situation de travail, ce qui est important et investi de valeur est étroitement articulé avec les règles de métier et la professionnalité, à ce qui a de la valeur aux yeux des personnes qui travaillent, à ce que signifie "bien travailler» ou "saboter le travail» (Clot, 2010). Dans ce sens, la question de la reconnaissance n'est pas tant celle de travailler pour être reconnu que de se reconnaître dans ce qu'on fait (Molinier, 2011, p. 17).

\subsubsection{Les enseignants du second degré et les doctorants : des risques liés au management et aux relations de travail}

Les enseignants du second degré et les doctorants - de même de manière massive les personnels administratifs et techniques toutes catégories ${ }^{1}$, dont nous ne traitons pas ici - évoquent largement les questions liées au management et aux relations de travail. Les sources de tensions évoquées sont ici concentrées sur les défaillances du collectif de travail, que ce soit en termes de répartition inéquitable des tâches qu'en termes d'absence de régulation des dysfonctionnements. Les enseignants étant amenés dans le système universitaire à prendre des responsabilités d'encadrement (par élection par leurs pairs) sans y avoir été préparés, les conflits inhérents à toute activité collective de travail les laissent démunis. Si des tensions avec les étudiants sont aussi

1. En revanche, le profil des facteurs de risques des personnels de direction s'apparente davantage à celui des enseignants-chercheurs, ce qui s'explique notamment par les responsabilités accrues depuis l'autonomie des universités. 
énoncées (incivilités, manque de discipline, agressivité, menaces, problèmes de santé), elles trouvent fréquemment un règlement ; en revanche, ce qui semble préoccuper les personnes relève de questions relatives à leurs partenaires dans l'activité.

\section{Tableau 4 : problèmes concernant le management et les relations de travail}

\begin{tabular}{|c|c|}
\hline $\begin{array}{l}\text { Hiérarchie, encadrement } \\
\text { Difficultés liées au management des } \\
\text { équipes }\end{array}$ & $\begin{array}{l}\text { - attentes excessives vis-à-vis de certains collègues (faire appel aux bonnes volontés) et } \\
\text { pas à d'autres (non impliqués) ; } \\
\text { - augmentation des conflits au sein des équipes (manque de mobilité des équipes); } \\
\text { - conscience d'un manque de formation en termes de management ; } \\
\text { - difficultés liées au positionnement (à l'interface de différents acteurs); } \\
\text { - difficultés liées aux différents statuts des personnels; } \\
\text { - leviers incitatifs et coercitifs faibles / collègues pour l'exercice de l'autorité (valorisation } \\
\text { du travail, sanctions, etc.) ; conséquences : des cas particuliers qui servent de paravent au } \\
\text { non engagement d'autres personnels ; devoir être en permanence dans la négociation ; } \\
\text { - manque de sanctions en cas de comportements déviants (procédures trop lourdes; } \\
\text { impunité). }\end{array}$ \\
\hline $\begin{array}{l}\text { Relations } \\
\text { Tensions, conflits avec les collègues }\end{array}$ & $\begin{array}{l}\text { - conflits entre collègues; } \\
\text { - anciens étudiants non intégrés en tant que collègues; } \\
\text { - collègue manipulateur ; } \\
\text { - divergences d'opinion, de positionnement sur certains sujets; } \\
\text { - manque de respect; } \\
\text { - rivalités, manque de soutien des collègues; } \\
\text { - tensions, clivages au sein d'une équipe; } \\
\text { - non-respect des locaux communs (bureaux, salles) / propreté ; } \\
\text { - problèmes de rapports de pouvoir (entre différentes catégories); } \\
\text { - abus de pouvoir / personnel ou /étudiants (exigences abusives, notation trop stricte, } \\
\text { intimidations, mépris, racisme); } \\
\text { - concurrence, conflits entre équipes ou filières; } \\
\text { - tensions, conflits causés par des égos surdimensionnés; } \\
\text { - tensions, conflits amplifiés par l'informatique (personnes ne se rencontrent pas pour } \\
\text { déminer les tensions). }\end{array}$ \\
\hline $\begin{array}{l}\text { Relations } \\
\text { Répartition non équitable de la charge } \\
\text { de travail ou des responsabilités }\end{array}$ & $\begin{array}{l}\text { - inadéquation entre le niveau de responsabilités et les compétences; } \\
\text { - devoir pallier aux manques des collègues; } \\
\text { - manque d'équité par rapport à la distribution des tâches administratives; } \\
\text { - répartition non équitable des heures d'enseignement. }\end{array}$ \\
\hline
\end{tabular}

Source : Durat L., Brunet F. (2013), Rapport RPS au CHSCT.

Les tensions professionnelles qui ne trouvent pas de règlement jugé équitable, d'étayage collectif, de soutien par les pairs, présentent les plus forts risques de dégradation en conflits relationnels durables. Ces situations sont repérables lorsqu'elles ont des effets sur la santé des personnes concernées, ce qui rend leur résolution longue et complexe.

Enfin, des interrogations, des inquiétudes et des regrets sont exprimés quant au manque de visibilité sur les évolutions à venir de l'enseignement supérieur et de la recherche, sur le 
périmètre jugé trop incertain des établissements, sur les pressions liées à la recherche permanente de financements extérieurs (par appel à projets, par sollicitation des collectivités territoriales) mais aussi sur les économies au sein des établissements (diminution des réserves de fonctionnement, suppression de filières à faible effectif, mutualisation de moyens). Des remises en question se font jour également concernant l'absence de latitude décisionnelle, la crainte d'un alourdissement du fonctionnement et d'une déshumanisation de la gestion des personnels.

\section{Conclusion}

A la lumière de ces résultats, nous souhaitons revenir sur un des écueils courants qui tient à la délicate prise en compte des maux du travail. Si les troubles sont exprimés par les individus, les risques professionnels liés au travail restent déterminés par l'environnement de travail dans ses diverses dimensions. Pour autant, on ne peut pas dire que les conditions du travail se soient dégradées (thème de l'entrée en décadence du travail, Dejours, 2008, p. 12) ni que la « souffrance au travail ", un affect déstabilisant du vécu psychique au travail, ait augmenté, même si on observe un accroissement des contraintes. Les expressions de stress, démotivation, souffrance et mal-être échappent à une causalité simple : " Ce ne sont pas tant les conditions de travail qui changent que la façon dont elles sont régulées, vécues et étiquetées" (Loriol, in Lallement \& al. 2011, p. 10). Ainsi, les tensions évoquées sont d'autant plus pathogènes qu'elles sont associées à une impuissance d'agir.

La capacité sociale des groupes à se saisir de ce qui fait problème contraste avec les situations où ces problèmes ne sont pas identifiés, sont déniés, minimisés, et ne peuvent alors trouver d'issue objective. Les répercussions sur la santé individuelle des personnes sont renforcées par ce renvoi à leur subjectivité - conçue comme les émotions et affects qui travaillent les sujets, à la racine même de l'activité productive et de la motivation - et produisent aussi bien le plaisir à travailler que potentiellement la souffrance au travail.

Alors "qui faut-il soigner: les travailleurs ou le travail?" pouvons-nous nous demander avec Lallement et Marry (2011, p. 6). Des raisons de satisfaction évoquées dans notre enquête apparaissent aussi, par exemple l'autonomie dans l'organisation du travail et les relations avec des personnes intéressantes (étudiants et collègues), le sentiment de faire œuvre utile au service de l'intérêt commun. Des propositions d'améliorations côtoient des réitérations de l'investissement et de la conscience professionnelle des personnes rencontrées. Cet engagement vers l'amélioration des processus organisationnels fait pencher la balance du côté du développement plutôt que de l'empêchement, des tensions avérées.

De nombreuses enquêtes ont montré que des filtres existent entre l'émergence de situations critiques et leur somatisation chez les personnes : le soutien des pairs dans le travail et hors travail, les réseaux sociaux (amicaux et familiaux), les stratégies de préservation de soi (retrait, évitement, mobilité) ou de protestation collective permettent une médiation ou la 
régulation de la vie au travail. Ces médiations entre organisation du travail et pathologies paraissent une voie prometteuse de la littérature sur les risques psycho-sociaux.

Un certain nombre d'auteurs poursuit cette ligne. Loriol (2012) notamment, dans ses travaux sur différents groupes professionnels, montre par exemple que les brigades où les policiers expriment le moins de stress sont celles qui réalisent tout un travail collectif de partage des connaissances sur les difficultés du terrain, d'élaboration en commun du sens des actions réalisées, de mise en place de routines d'action, de retour d'expérience sur les interventions, de gestion précoce des conflits avec les usagers et de soutien mutuel. De même, Richard (2012) soutient que la construction et l'animation d'espaces de discussion par les organisations constituent un puissant levier de préservation des ressources (compétences, règles de métier, capacités, pouvoir d'agir, identité professionnelle...) et donc un facteur de bien-être - et de qualité - au travail.

Notre hypothèse est que dans le domaine de l'enseignement supérieur et de la recherche, les contraintes accrues et les transformations du travail se sont ajoutées à une fragilité des collectifs de travail ; cette accumulation produit souffrance, mal-être et stress.

Dans les universités, le temps de co-présence entre collègues est faible, la possibilité de s'ajuster collectivement aux situations trop rare et non valorisée par l'institution ${ }^{2}$. L'autorégulation individuelle ne suffit plus, parce qu'elle fait du professionnel la variable d'ajustement d'un système de règles formelles et impersonnelles, de contrôle a posteriori : «Le salarié qui se retrouve seul à gérer des difficultés qui le dépassent est réduit à faire valoir son stress pour rappeler des efforts devenus invisibles" (Loriol, in Lallement \& al. 2011, p. 13). C'est à travers la coopération basée sur des règles, des valeurs et des expériences partagées par les groupes de travail, ou ce que Reynaud (1989) appelle "les régulations autonomes ", que Clot (2010) nomme "la dispute professionnelle " au sens de délibération sur le sens et la qualité du travail et de professionnalité, ou encore Schwartz (2001), le " débat sur les normes antécédentes", que les personnes peuvent ressaisir les contradictions, tensions, controverses qui tissent le travail. Dans la saisie des situations par le travail collectif, l'occasion d'une délibération sur les fins et valeurs de l'activité permet de prendre en charge les contradictions, les antagonismes qui surviennent au cours de l'action : «Si les régulations et marges de manoeuvre collectives ne parviennent pas à aider les opérateurs à gérer les conflits de buts, on passe d'une situation épineuse ou conflictuelle à une véritable situation critique qui devient source de stress pour les individus. Le care n'est pas qu'une affaire individuelle, il repose aussi sur les possibilités ouvertes par le collectif de travail» selon Caroly (2010, p. 222).

L'université doit rendre possibles ces espaces d'échange sur les pratiques, ces communautés discursives, pour la vitalité des collectifs de travail et la santé des individus. A ce prix, le travail, qui représente toujours un risque psychosocial puisque l'on s'y engage soi-même (Molinié, 2011, p. 16), peut être à la fois une activité productive et constructive.

2. Seule la présence aux jurys est obligatoire, les réunions d'équipes pédagogiques sont à géométrie variable. 


\section{- Bibliographie}

Abord de Chatillon E. \& Desmarais C. (2012), « Le Nouveau Management Public est-il pathogène ?" Management international, 16(3), pp. 10-24.

Baudelot C., Gollac M., Bessière C., Coutant I. \& Godechot O. (2003), Travailler pour être heureux?, Le bonheur et le travail en France, Paris, France, Fayard.

Caroly S. (2010), L'activité collective et la réélaboration des règles : des enjeux pour la santé au travail. Note de syntèse HDR, Bordeaux, Université Victor Segalen.

Clot Y. (2010), Le travail à cour : pour en finir avec les risques psychosociaux, Paris, France, La Découverte.

Dejours C. (2008), Travail, usure mentale: essai de psychopathologie du travail. Paris, France, Bayard.

Dejours C. \& Groupe Sciences en questions (2003), L'évaluation du travail à l'épreuve du réel : critique des fondements de l'evaluation, Paris, Institut national de la recherche agronomique.

Durat L., Brunet F. (2013), Evaluation des Risques psycho-sociaux du personnel, Rapport à destination des instances du CHSCT de l'UHA.

Froment E. (2012), La technique des focus groups dans la l'analyse des risques psychosociaux, CNAM, Centre régional (Champagne-Ardennes), Reims, France.

Gollac M. \& Volkoff S. (2007), Les conditions de travail, Paris, France, La Découverte.

Gollac M., Bodier M. (dir.) (2011), Mesurer les facteurs psychosociaux de risque au travail pour les maitriser, DARES, ministère du Travail et de l'Emploi.

Grosjean V. \& Institut national de recherche et de sécurité (2004), Le bien-être et la santé au travail : position du problème, Paris, Edition INRS.

Hirigoyen M.-F. (1998), Le harcèlement moral : la violence perverse au quotidien, Paris, France, Presses Pocket.

Hirigoyen M.-F. (2001), Malaise dans le travail : harcèlement moral, démêler le vrai du faux. Paris, le Grand livre du mois.

Hirigoyen M.-F. (2002), Le harcèlement moral dans la vie professionnelle : démêler le vrai du faux, Paris, France, Pocket.

Institut national de recherche et de sécurité (2010), Dépister les risques psychosociaux: des indicateurs pour vous guider, Paris, France, INRS.

Karasek R. \& Theorell T. (1990), Healthy work: stress, productivity, and the reconstruction of working life. New York, Etats-Unis: Basic Books. 
Lallement M., Marry C. \& Loriol M. (2011), « Maux du travail: dégradation, recomposition ou illusion?", in Sociologie du travail, Issy-les-Moulineaux, France, Elsevier Masson, pp. 3-36.

Lhuilier D. (2012), Cliniques du travail, Toulouse, France, ERES.

Loriol M. (2000), Le temps de la fatigue : la gestion sociale du mal-être au travail, Paris, France, Anthropos, Economica.

Loriol M. (2012), La construction du social. Souffrance, travail et catégorisation des usagers dans l'action publique, Presses Universitaires de Rennes.

Musselin C. (2009), "Les réformes des universités en Europe : des orientations comparables, mais des déclinaisons nationales ", Revue du MAUSS, n 33(1), pp. 69-91.

Reynaud J.-D. (1989), Les règles du jeu : l'action collective et la régulation sociale, Paris, France, A. Colin.

Richard D. (2012), Management des risques psychosociaux : une perspective en termes de bienêtre au travail et de valorisation des espaces de discussion, Thèse de doctorat, Institut de recherche en gestion et économie, Annecy.

Robert N., Grosjean V. \& Institut national de recherche et de sécurité (2006), Développement d'un questionnaire orienté bien-être : pour un dialogue renforcé Médecine du travail Ressources humaines, Paris, France, Editions INRS.

Sahler B., Berthet M., Douillet P., Mary-Cheray I. \& Agence nationale pour l'amélioration des conditions de travail (2007), Prévenir le stress et les risques psychosociaux au travail, Lyon, France, ANACT.

Schwartz Y. (2001), Le paradigme ergologique ou un métier de philosophe, Toulouse, France, Octares.

Siegrist J., Starke D., Chandola T., Godin I., Marmot M., Niedhammer I. \& Peter R. (2004), "The measurement of effort-reward imbalance at work: European comparisons", Social science \& medicine (1982), 58(8), pp. 1483-1499.

SUMER (1994, 2003, 2010), Expositions aux contraintes et nuisances dans le travail, résultats de l'enquête Surveillance médicale des risques, Paris, ministère de l'Emploi et de la Solidarité, La Documentation française.

Van Belleghem L., De Gasparo S. \& Gaillard I. (2013), «Développement de la dimension psychosociale au travail ", in Falzon P. (dir.) Ergonomie constructive, Paris, PUF.

Viry L. (2006), Le monde vécu des universitaires, ou la République des Egos, Rennes, PUR.

Volkoff S. (2013), Les facteurs de risques psychosociaux : leurs formes et leurs conséquences, Bivi AFNOR. Consultable à l'adresse : http://www.bivi.maitrise-risques.afnor. org/ofm/maitrise-des-risques/v/v-10/v-10-90/1 